\title{
Comparative Analysis of Crack Resistance of Fiber Metal Laminates with HS2 Glass/T700 Carbon Layers for Various Stress Ratios
}

\author{
X. Song, ${ }^{\text {a,1 }}$ Z. Y. Li, ${ }^{a}$ Y. Shen, ${ }^{a}$ Y. L. Chen, ${ }^{a}$ and J. Z. Zhang, \\ a School of Mechanical and Power Engineering, Harbin University of Science and Technology, \\ Harbin, China \\ ${ }^{\mathrm{b}}$ Composite Research and Structure Centre, Harbin Institute of Technology, Harbin, China \\ ${ }^{1}$ songxin121@sina.com
}

Because of good crack growth resistance owe to the fibers between the aluminum layers, fiber metal laminates (FML) offer significant improvements compared to currently applied materials for aircraft structures. The crack resistance of different reinforced fibers, glass fiber, and carbon fiber, under stress ratio $R=-1,0.1$, and 0.5, respectively, are analyzed through test data. The results showed significant scatter in the positive stress ratio and obvious difference between two outer aluminum layers. So, the failure criterions of metal materials specified in the test standard are unsuitable for the FMLs fatigue test, and it is a problem to determine the failure criterion according to the engineering applications. This study is an experimental investigations with respect to the FMLs crack resistance and a qualitative conclusions have been drawn from the data analysis.

Keywords: fiber metal laminates, glass fiber, carbon fiber, crack resistance, failure criterion.

Introduction. Fiber metal laminates (FML), as an interplay hybrid composite, is composed of metal lamina and reinforced fibers that are sticked layer by layer, and then, solidified under proper temperature and pressure [1]. FMLs has the characteristics of metal and fiber composite materials, especially, the high resistance against crack growth. Furthermore, with the emergence of high strength alloy and new composite materials, the study of FMLs properties, which are composed of different new materials, have been developed [2]. New style FMLs will be studied for remarkable properties improvements, such as, light weight, high anti-fatigue properties and vibrations resistance, high specific strength and specific stiffness, good toughness, and machinability, etc. [3]. These superior properties make it very suitable for the new generation airplane structures [4]. So, the crack resistance of new style FMLs must be studied deeply especially. There are some finite element research to analyze mechanical and crack resistance of FMLs [5, 6]. In the earlier studies, fracture behavior of FMLs have been simulated by FE method [7, 8]. In this paper, the crack resistance of FMLs with different reinforced fiber, glass fiber and carbon fiber, under stress ratio $R=-1,0.1$, and 0.5 , are analyzed by test data. For the reason of significant scatter in the positive stress ratio, and obvious difference between two outer aluminum layers, a qualitative analysis is implemented.

1. FMLs Specimen Preparation. Composed materials of FMLs specimens are, in this paper, LY12M aluminum alloy layers with $1 \mathrm{~mm}$ thickness, HS2 glass fiber prepreg layers (GFML) and T700 carbon fiber prepreg layers (CFML), and sticked by J272 middle-temperature-setting-glue. The interlayes of aluminum alloy are bonded with fiber on the double side; outer layers of aluminum alloy are bonded on the single side. The surface of aluminum alloy layers should be treated by the method of phosphoric acid anodizing, according to the standard HB/Z197-91. Ambient temperature of specimen preparation is $25 \pm 5^{\circ} \mathrm{C}$ relative humidity $\leq 65 \%$. Solidification temperature is $120-125^{\circ} \mathrm{C}$, pressure is $0.2-0.4 \mathrm{MPa}$. After solidification process, the quality of FMLs must be checked by the nondestructive testing (NDT) technology. Figure 1 shown the NDT results of different solidified process with ultrasonic C-scan system. Light colour area is defective 
region (Fig. 1a) and deep colour area is the qualified solidifying portion of FMLs (Fig. 1b). Figure 2 showed the fractography analysis of FMLs with weak and good solidifying quality, respectively. In the defective region, delaminating (Fig. 2a) and bubbles (Fig. 2b) may exist. Fractography analysis of good solidifying quality is presented in Fig. 2c.

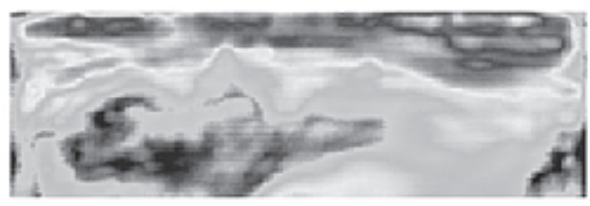

a
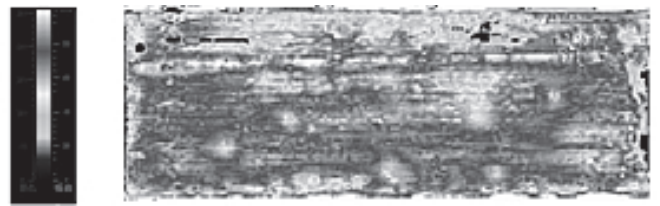

b

Fig. 1. Different results of FMLs with different thermo-compression solidification process [(a) have more defects than (b)].

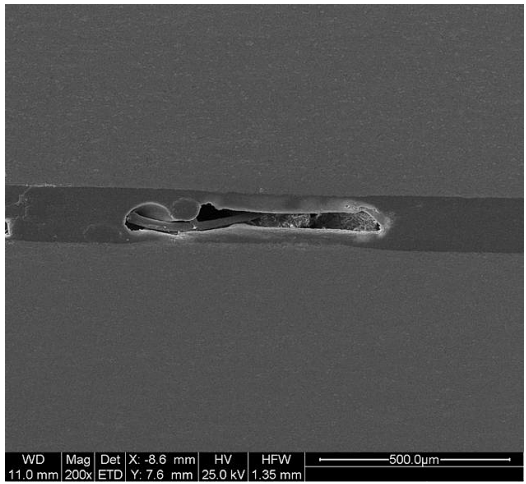

a

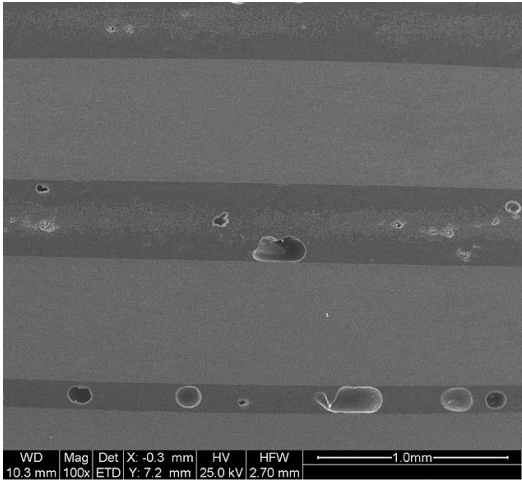

b

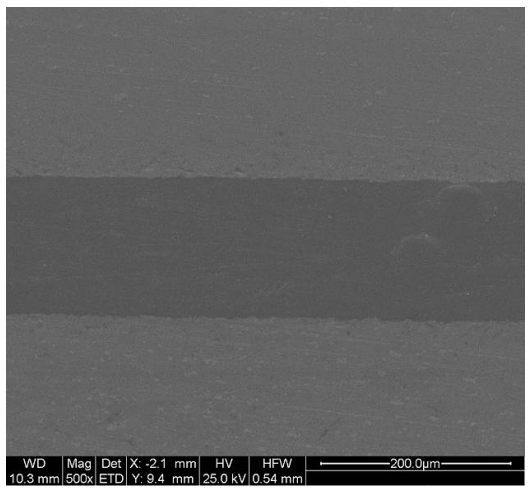

c

Fig. 2. Fractography analysis of differnet solidifying quality: (a) delaminating in $\times 200$ magnifying; (b) the composites bubbles in $\times 100$ magnifying; (c) fractography in $\times 500$ magnifying.

2. Fatigue Test of FMLs Specimen. Fatigue crack propagation rate test have done according to ASTM E-647, figuration of specimen shown in Fig. 3, fatigue crack prepare and fatigue crack propagation test implemented by PLG-100C high-cycle fatigue-testing machine (Fig. 4), loading orientation of uniaxial constant amplitude load along the fiber lay-up direction, stress ratios $R$ are $-1,0.1$, and 0.5 , respectively. Normally, stiffness failure criterion is used in fatigue test of composite material. During the fatigue test, with the damage accumulation rising, resonance frequency of the system comprised by fatigue test machine and specimen together is descending gradually. In this study, test is finished 


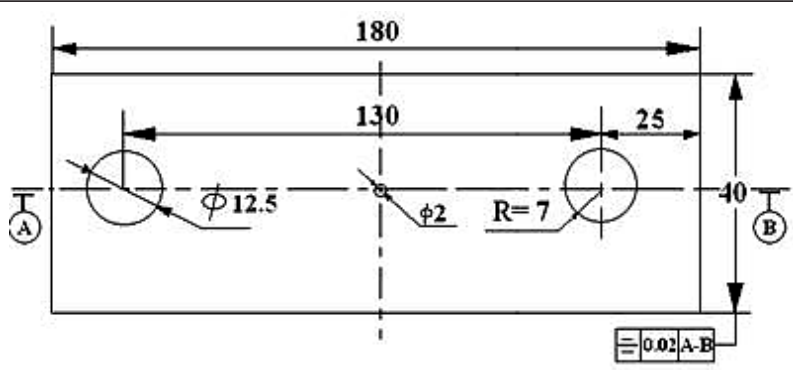

Fig. 3. LY12M aluminum alloy specimen configuration.

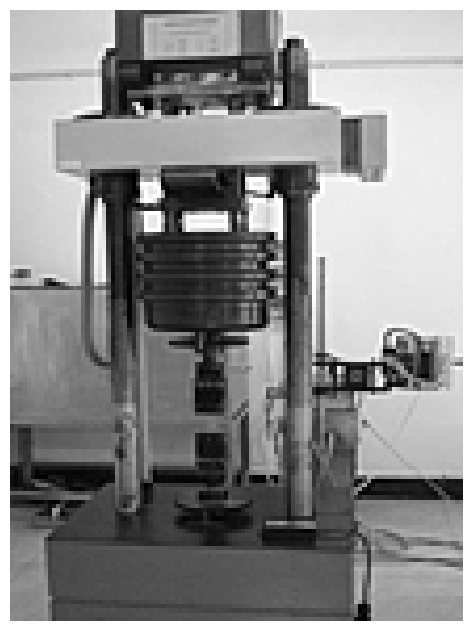

Fig. 4. PLG-100C high-cycle fatigue-testing machine.

when the first machine halt occurred which is triggered by the insufficient stiffness of specimen. Crack propagation data are acquired by the specimen photograph after assigned loading cycle, measured by image processing software programmed in Adobe Flash.

For the metal material specimen, test data are invalid if crack sizes measured on front and back surfaces differ by more than $0.25 B$ ( $B$ is the width of specimen). As a kind of composite material, fatigue damage forms of FMLs are different from other metal. Not only the performance are different between each layer of FMLs, but also there are residual stress will exist in specimen after solidification and machining process [9], as a result, it makes a significant difference between two outer metal laminates in fatigue crack growth, as shown in Fig. 5. Nowadays, there are no reasonable ways to determine the crack length of FMLs specimen when the large diversity occurred. In this paper, crack lengths of two outer metal laminates, at the same time, are all recorded. Because during the test, there has emerged through crack of one side, the other not, while the test still go on. Therefore, the numbers of records two of outer surfaces are different. The number of specimen tested and test data recorded for different test are listed in Table 1.

3. Fatigue Test Results Analysis. In the Paris law, $d a / d N=C(\Delta K)^{m}$, fatigue crack growth rate $d a / d N$ is the function of stress intensity factor range $\Delta K$ (or maximum stress intensity factor $K_{\max }$ when stress ratio $R$ is negative.). The relationship is usually shown with empirical curve fitting curves. Take logarithm on both sides of the Paris formula, $\log (d a / d N)=\log C+m \log (\Delta K)$, in the double logarithmic coordinates, with a set of $\log (\Delta K)$ and $\log (d a / d N)$, material constant $C$ and $m$ can be determined by the test data linear fitting curve. Value of $\Delta K$ is calculated by the method specified in the ASTM E647. 
Comparative Analysis of Crack Resistance of Fiber Metal Laminates ...

$\mathrm{T}$ a b 1 e 1

Number of Specimes Tested and Test Data Recorded for Different Test

\begin{tabular}{|c|c|c|c|c|c|c|c|c|}
\hline \multirow{3}{*}{$\begin{array}{c}\text { Stress } \\
\text { ratio } \\
R\end{array}$} & \multicolumn{8}{|c|}{ Material } \\
\hline & \multicolumn{3}{|c|}{ CFML } & \multicolumn{3}{|c|}{ GFML } & \multicolumn{2}{|c|}{ LY12M } \\
\hline & NST & TDRF & TDRB & NST & TDRF & TDRB & NST & TDR \\
\hline-1 & 8 & 70 & 107 & 7 & 61 & 47 & 9 & 100 \\
\hline 0.1 & 5 & 39 & 67 & 7 & 66 & 66 & 7 & 58 \\
\hline 0.5 & 7 & 91 & 96 & 6 & 39 & 39 & 9 & 68 \\
\hline
\end{tabular}

Note: NST correspond to number of specimens tested; TDRF - test data recorded of front surface; TDRB - test data recorded of back surface.

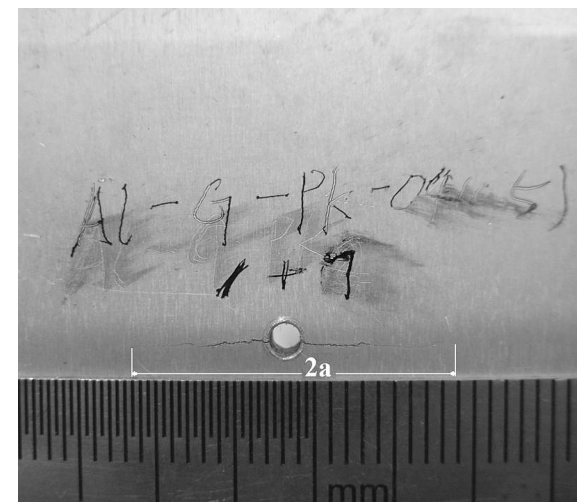

a

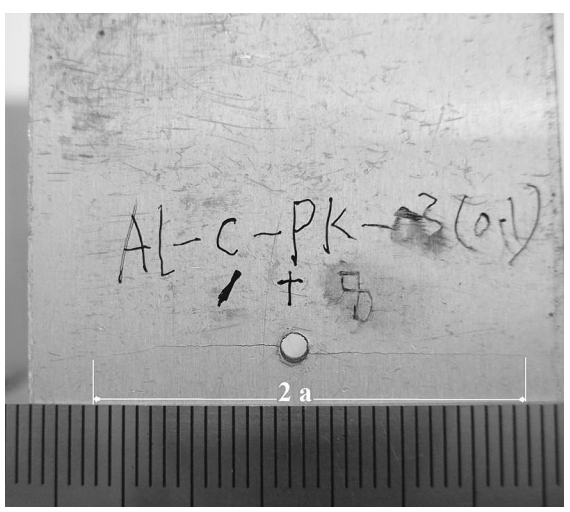

$\mathrm{c}$

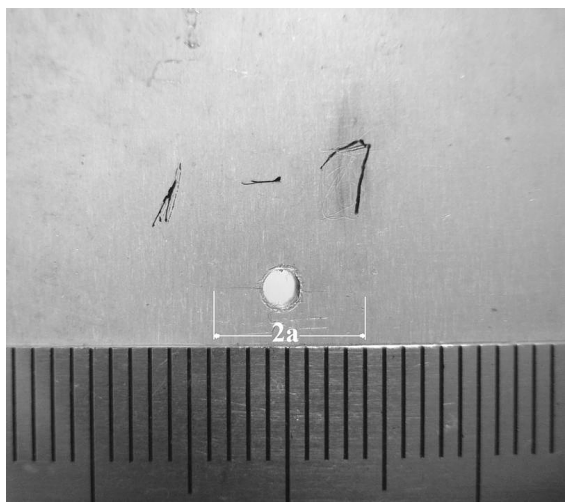

b

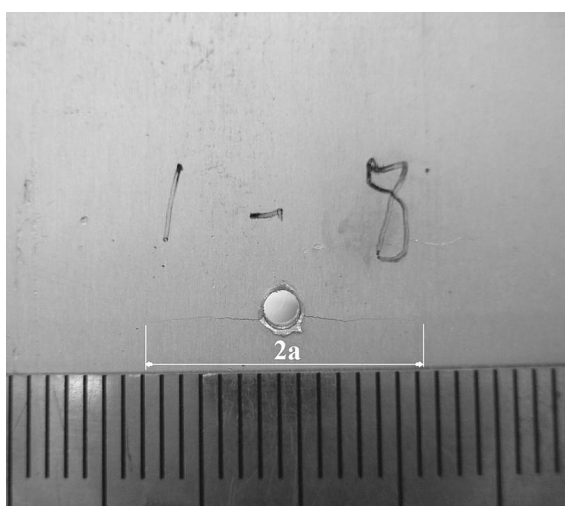

d

Fig. 5. Different fatigue crack propagation performance of two outer metal layers: front (a) and back (b) of glass fiber reinforced metal laminates; front (c) and back (d) of carbon fiber reinforced metal laminates.

When the linear correlation factors, $R^{2}$, approaches 1 , it means the test data exhibit a linear correlation in present coordinate system, otherwise, it is implied that the test data scattered broadly. It can be seen that in Table $2, R^{2}$ of FMLs are very low, compared to reference specimens which are made from the identical material, LY12M aluminum alloy, so, it indicates that the FMLs test data are dispersed in a wide range. 
$\mathrm{T}$ a b 1 e 2

\section{Correlation Factor $\boldsymbol{R}^{2}$ for Different Test Data}

\begin{tabular}{||c|c|c|c|c|c||}
\hline \multirow{2}{*}{$\begin{array}{c}\text { Stress } \\
\text { ratio } \\
R\end{array}$} & \multicolumn{4}{|c|}{ Material } & \multirow{2}{*}{ LY12M } \\
\cline { 2 - 6 } & Front & Back & Front & Back & \\
\cline { 2 - 6 } & 0.13 & 0.07 & 0.45 & 0.17 & 0.80 \\
\hline-1 & 0.47 & 0.44 & 0.35 & 0.54 & 0.87 \\
\hline 0.1 & 0.26 & 0.36 & 0.59 & 0.44 & 0.86 \\
\hline 0.5 & & & & \\
\hline
\end{tabular}

A qualitative analysis of fatigue crack growth tendency of different fiber reinforced FMLs has been done. In Fig. 6, under different stress ratio $R$, LY12M specimen test results are presented, while the FMLs fitting curves are derived from arithmetic average of fatigue crack growth rate of FMLs specimen front and back surface. So, there is a big deviation in this simplified method.

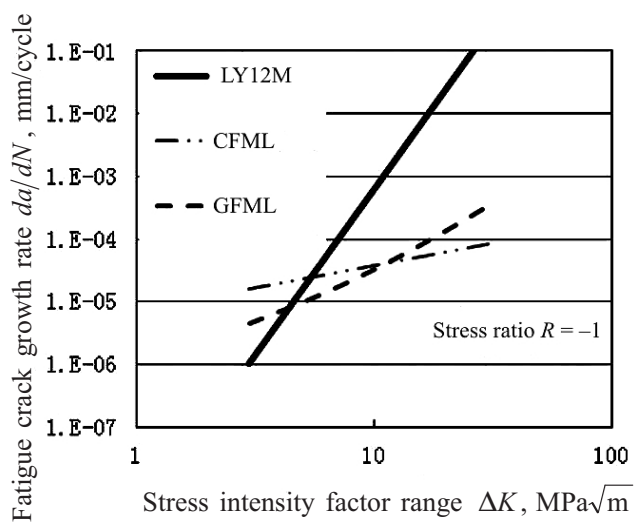

a

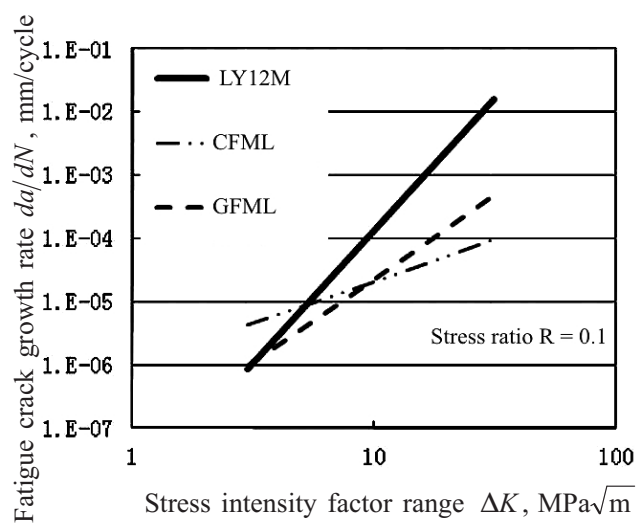

b

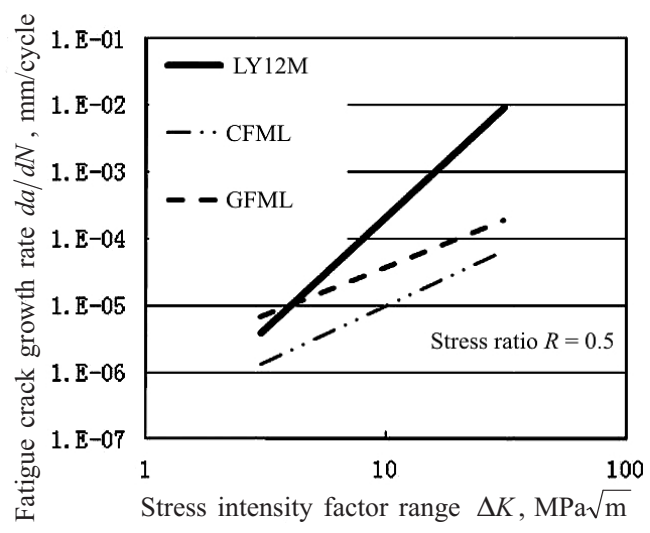

c

Fig. 6. Comparison of different fatigue crack propagation performance under different stress ratio $R$.

From Fig. 6a, it can be seen that in the initial stage of crack propagation, $d a / d N<10^{-5} \mathrm{~mm} /$ cycle, because of more defects in FMLs than that in identical material specimen, the effect of compressive stress promote damage increasingly more in composites 
than in metal, consequently, $d a / d N$ of FMLs are higher than which of LY12M specimen. However, in high crack growth rate region, $d a / d N>10^{-4} \mathrm{~mm} /$ cycle, because of the bridge linkage of reinforced fiber, $d a / d N$ of FMLs are lower than which of LY12M specimen. This feature is also shown in the case of positive $R$, Fig. $6 \mathrm{~b}$ and $6 \mathrm{c}$. With the stress range decreasing, as listed in Table 3, different tension strength of components, $\sigma_{b}$ of T700 is about $4900 \mathrm{MPa}$ and of HS2 is about $4278 \mathrm{MPa}$, effects on crack growth differently. When $R=0.5$, T700 carbon fiber FMLs specimen have lowest crack growth rate, LY12M specimen have fastest rate, accordingly, carbon fiber FMLs specimen have the largest tension strength, and LY12M specimen have least strength.

$\mathrm{T}$ a b 1 e 3

Test Load for Different Stress Ratio

\begin{tabular}{|c|c|c|c|c|c|c||}
\hline \multirow{2}{*}{$\begin{array}{c}\text { Stress } \\
\text { ratio } \\
R\end{array}$} & \multicolumn{4}{|c|}{ Material } \\
\cline { 2 - 7 } & \multicolumn{2}{|c|}{ CFML } & \multicolumn{2}{c|}{ GFML } & \multicolumn{2}{c|}{ LY12M } \\
\cline { 2 - 7 } & $P_{m}, \mathrm{kN}$ & $\Delta P, \mathrm{kN}$ & $P_{m}, \mathrm{kN}$ & $\Delta P, \mathrm{kN}$ & $P_{m}, \mathrm{kN}$ & $\Delta P, \mathrm{kN}$ \\
\hline 0.1 & 0.47 & 0.44 & 0.35 & 0.54 & 3.51 & 2.87 \\
\hline 0.5 & 0.26 & 0.36 & 0.59 & 0.44 & 7.50 & 2.50 \\
\hline
\end{tabular}

4. Concluding Remarks. It is unreasonable in FMLs fatigue test when the strength failure criterions are used as identical metal material test or nonmetal composites test. Because the defects and residual stress are inevitable after specimen preparation, and fatigue crack growth behavior between two outer metal laminates of FMLs specimen is different, it is proper to record all these two fatigue data and analyze respectively. It is suitable to determine the test end condition according to stiffness failure criterion, that is test finished when the first machine halt occurred because of the insufficient stiffness of specimen.

In the select-reinforced materials study, tension strength of different reinforced fiber affect fatigue cracks propagation rate for the main reason of bridge linkage phenomenon around crack tip zone, especially, in the case of small stress amplitude range applied. For fiber reinforced composites specimen the compressive stress will promote crack propagation, so, when stress ratio $R$ is negative, compressive stress in fatigue loading has more effect on $d a / d N$ of FMLs specimens in the initial stage of crack propagation.

Acknowledgments. The work was supported by the National Nature Science Foundation of China under the Contract No. 51271067.

1. X. R. Wu and Y. J. Guo, "Fatigue life prediction of fiber reinforced metal laminates under variable amplitude loading," Eng. Sci., 1, No. 3, 36-39 (1999).

2. M. Boscolo, G. Allegri, and X. Zhang, "Design and modeling of selective reinforcements for integral aircraft structure," AIAA J., 46, No. 9, 2323-2331 (2008).

3. J. Bienias, B. Surowska, and P. Jakubczak, "The comparison of low velocity impact resistance of aluminum/carbon and glass fiber metal laminates," Polym. Compos., Doi: 10.1002/pc.23266 (2014).

4. J. F. Laliberté, C. Poon, P. V. Straznicky, and A. Fahr, "Applications of fiber-metal laminates," Polym. Compos., 21, No. 4, 558-567 (2000).

5. M. Grassi and X. Zhang, "Finite element analyses of mode I interlaminar delamination in z-fibre reinforced composite laminates," Compos. Sci. Technol., 63, 1815-1832 (2003). 
6. Y.-Q. Liao, J.-H. Su, and S.-L. Ke, "Application of ANSYS in composite simulation analyses," Fiber Compos., 23, No. 4, 63-66 (2006).

7. X. Song and J. Z. Zhang, "Analysis of a finite element model of 2-D elastic-plastic crack," J. Harbin Univ. Sci. Tech., 13, No. 5, 9-13 (2008).

8. X. Song, Z. Liu, and J. Z. Zhang, "Simulation study of fracture behavior in a FMLs specimen by 3D elastic-plastic finite element model," Polym. Polym. Compos., 19, No. 2-3, 209-212 (2011).

9. G. T. Zhang, W. G. Chen, and B. Yang, "Testing research on mechanical properties of T700 carbon fiber/epoxy composites," Fiber Compos., 49, No. 2, 49-52 (2009).

Received 03. 08. 2015 